\title{
Preliminary Studies on Transition Curve Geometry: Reality and Virtual Reality
}

\author{
Tanita Fossli Brustad ${ }^{a^{*}}$ \\ ${ }^{a}$ Department of Computer Science and Computational Engineering, UiT The Arctic University of Norway, Narvik, Norway
}

\begin{abstract}
The topic of transition curves is well known in track and road engineering. Over the years the need for easement between straight and curved sections in railway and road design has grown, with an important reason being increase in vehicle speed. Testing of transitions is often done with graph analysis where the property of lateral change of acceleration is compared between varieties of curves. However, graph analysis does not give a clear understanding of the behaviour of a vehicle in a transition phase the way, for instance, field or laboratory experiments might do. In this paper we present an initial study on the behaviour of a down-scaled vehicle model driving through three distinct transitions from straight to curved sections. In addition we show a comparison of the physical model against a virtual model.
\end{abstract}

Keywords:

Geometry; Transition Curves; Lateral Change of Acceleration; Curvature; Virtual Reality.

\section{Article History:}

$\begin{array}{llll}\text { Received: } & 13 & \text { December } & 2019 \\ \text { Accepted: } & 26 & \text { January } & 2020\end{array}$

\section{1- Introduction}

Horizontal geometry has been, and is, a popular topic in railway and road design. One focus area is the transition curve and the properties this curve should have. Transition curves are used as easement between straight sections and curved section in track and road engineering [1, 2]. Over the years different criteria have laid the foundation when deciding the type of curve. In the beginning no transitions were needed because of low speed and wide-radius curves, however during the 19th century when the vehicle speed increased the need for easement arose and the clothoid (also referred to as Euler spiral or Cornu spiral) became the most popular [3]. The clothoid is still the most used transition curve today, but with the ever increasing speeds and with the introduction of lateral change of acceleration (LCA) [4] as the most important criterion in vehicle-road dynamics [5], other curves have been recommended in order to increase safety and comfort, and to reduce the maintenance need. The way of testing the properties of a transition curve nowadays is mostly done by considering a graph of the LCA function, and comparing it to the LCA of the clothoid, as in [6, 7]. Field and laboratory testing on transition geometry are not very common in research, with a prominent reason being cost and time, but also because transition research has moved in a direction where a graph of the LCA function is enough information to categorize the quality of the curve. The motivation behind this paper is to increase the understanding of the effect of transition curves by studying the lateral forces working on the vehicle, and by observing the path traced by the vehicle for three different transitions from straight sections to curved sections, in a laboratory study setting. As an initial study and to easily be able to extract path data, a down scaled vehicle model will be used in the laboratory experiments. In addition, a comparison of the physical vehicle model against a virtual model will be given in order to increase the validity of the results and to test if simple VR technology resembles the physical model in behaviour.

In this paper we look at a simple physical vehicle model and examine what observable effects geometry has on a vehicle in a transition phase (going from a straight road into a turn). In addition we create a virtual model of the vehicle to investigate if existing straightforward desktop-based VR technology [8] can give a satisfactory result compared to the

\footnotetext{
* CONTACT: Tanita.f.brustad@uit.no

DOI: http://dx.doi.org/10.28991/esj-2020-01204
}

(C) 2019 by the authors. Licensee ESJ, Italy. This is an open access article under the terms and conditions of the Creative Commons Attribution (CC-BY) license (https://creativecommons.org/licenses/by/4.0/). 
physical model when it comes to the behaviour of the vehicle.

\section{2- Preliminaries}

This section gives a brief description on; which transition geometry we considered for the experiments, the technology to create the physical vehicle model (Lego Mindstorms), and the software used to create the virtual vehicle model (Blender).

\section{2-1- Transition Geometry}

There are many different curves suitable as transition curves. In railways, highways, and robot trajectory design it is desirable to have curves with monotone curvature [9]. As mentioned in the introduction the most commonly used in practice (especially in railway) is the clothoid. A clothoid curve (of length $l$ and end radius $r$ ) is defined parametrically, with Fresnel integrals, as

$x=C(t)=\frac{1}{a} \int_{0}^{\hat{t}} \cos \left(\frac{\pi}{2} u^{2}\right) d u$,

$y=S(t)=\frac{1}{a} \int_{0}^{\hat{t}} \sin \left(\frac{\pi}{2} u^{2}\right) d u$.

Where $a=\sqrt{\frac{1}{\pi r l}}$ is a scaling factor, and $\hat{t}=a t,-\infty<t<\infty$, see Figure 1 .

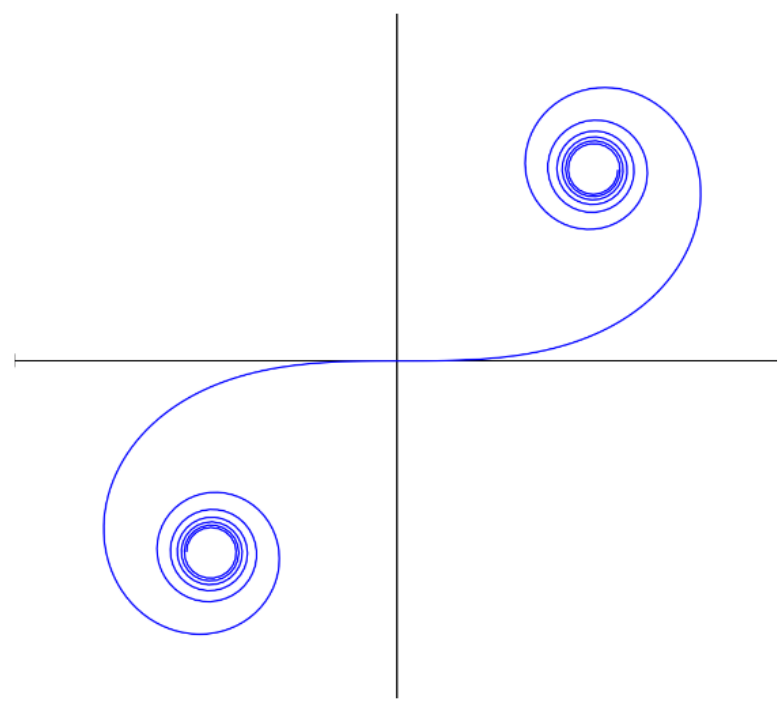

Figure 1. The clothoid spiral.

This particular curve is preferred in railway tracks because of some desired properties it possesses: the curvature changes linearly with curve length, and the curve is easy to scale, in relation to curve length and the radii in both ends. An example of the clothoid as a transition between a straight and a circular segment can be seen in Figure 2.

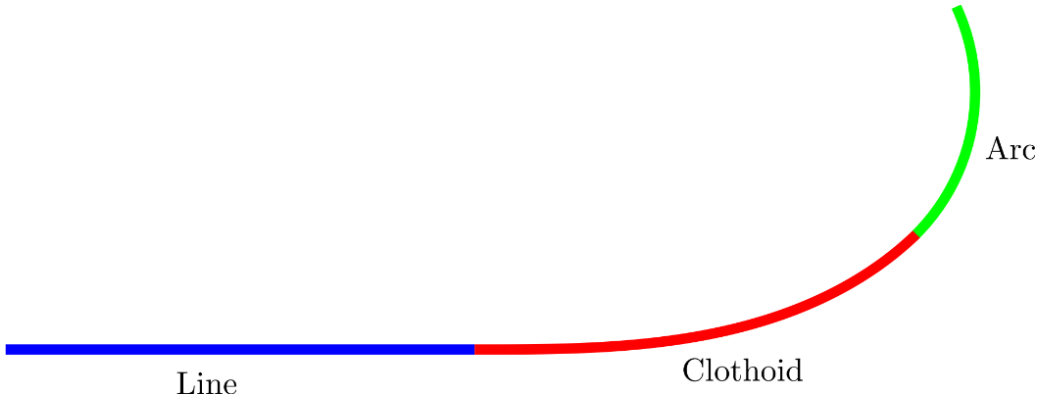

Figure 2. A straight curve and a circular curve connected by a clothoid transition curve.

Taking into account the LCA-function, given by [4];

$z=-\frac{p v}{\sqrt{u^{2}+p^{2}}}\left(3 k a_{t}+v^{2} \frac{d k}{d l}-\frac{k v^{2} u+g p}{u^{2}+p^{2}} \frac{d u}{d l}\right)$.

Where $p$ is the horizontal width between the wheels, $v$ is the velocity of the vehicle, $u$ is the superelevation, $k$ is the curvature along the curve, $a_{t}$ is the tangential acceleration, and $g$ is the gravity constant, the clothoid is not an optimal 
transition curve because of its $G^{0}$ continuity of the curvature in the joints. However, it is better than no transition curve which will cause a discontinuity in the curvature between segments. A more optimal transition curve would be one where the curvature has a higher geometrical continuity in the joints than $G^{0}, G^{n}, n>0$, while still being monotone and not too steep in the middle section. Ideally, a transition curve should have a curvature closely connected to a sigmoid function (the curvature should be S-shaped), in order to improve the LCA which is closely connected to the curvature. For the comparisons in this paper we take into account what we know about the LCA function and choose to look at three different scenarios: a track with no transition, a track with a clothoid transition, and a track with a transition that has an S-shaped curvature (we will call it an S-shaped transition). Figure 3 shows the curvature of the three transition cases.

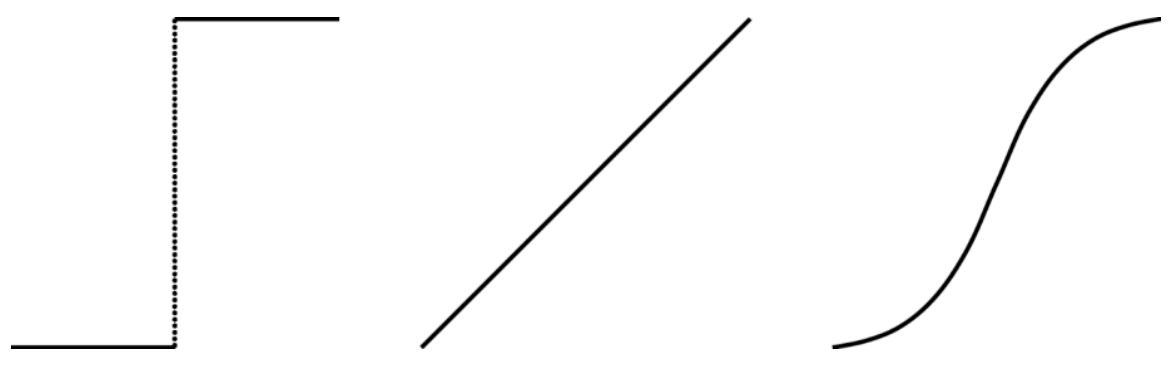

Figure 3. The curvature of the transition cases (Left: No transition curve. Middle: Clothoid transition curve. Right: Sshaped transition curve).

\section{2-2-Lego Mindstorms}

Lego Mindstorms [10] was launched in 1998 and is a software platform for programming Lego inspired robots. Each Mindstorms set consists of Lego building blocks, an intelligent brick that controls the system, and a set of sensors (including motors). Since the start there have been four generations of the Mindstorms platform with the latest being Lego Mindstorms EV3. Lego Mindstorms comes with its own programming software that uses a block-based drag and drop interface where blocks performing a certain task are linked to make the robot run as intended (Figure 4 shows a small example program). In addition many other programming languages are supported, both block-based and textbased.

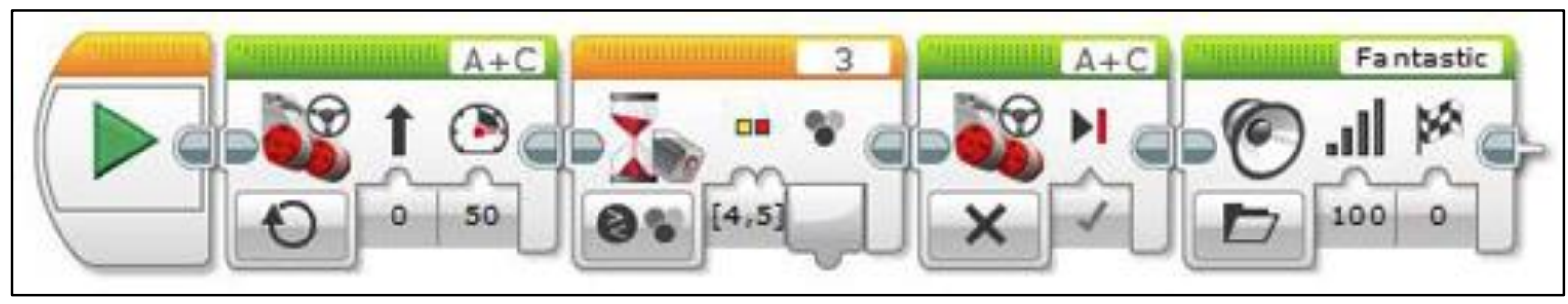

Figure 4. An EV3 software program that makes the robot drive forward until the colour red or yellow is seen. Then the robot stops and shouts "Fantastic".

The reason for choosing Lego Mindstorms as the test vehicle was mainly because of its small size, making it easy to work with, and because of the various sensors that are available for collecting a number of parameters (the most interesting to us was the gyroscope). We also concluded that a small model would have the same behaviour as a larger model in regards to a transition phase, especially if we consider the LCA function which only includes two parameters from the vehicle; velocity and tangential acceleration. So based on the fact that the Lego motors can maintain a stable constant maximum velocity when running, which means that the tangential acceleration is zero, the lateral forces and the traced path of this small model should, in theory, not differ much from a larger model running with constant speed.

\section{2-3-Blender Game Engine with Bullet Physics}

Blender is a 3D creation software with tools including modelling, rendering, rigging, simulations, and game creation [11]. The game creation tool, Blender Game Engine [12], can be used for real-time projects. The engine allows the creation of interactive 3D application or simulations, and oversees a game loop which processes logic, sound, physics and rendering simulations in sequential order. A number of powerful libraries are taken advantage of in the Game Engine, one of them is the Bullet Physics library [13]. This is a physics library that includes 3D collision detection, soft body dynamics, and rigid body dynamics.

The reason for choosing Blender to create a virtual model was because of its physics engine, and because Blender is a well-known tool. Blender offers a physics object called a Vehicle Controller [14] that the engine and Bullet recognize. The Vehicle Controller offers a stable vehicle simulation with simplified physics, that still acts as you would expect a physical vehicle to act, and provides the opportunity to tweak various parameters connected to the vehicle (i.e. maximum 
velocity). So with the same argumentation as with the Lego vehicle on LCA, the Blender model should, in theory, be suited for experimentation regarding lateral forces and traced paths since the model acts close to a physical vehicle and can hold a constant maximum speed.

\section{3- Method}

This section describes the setup of the physical and virtual models, how the experiments were created and performed, and how the resulting plots were visually compared.

\section{3-1-The Physical Model}

The physical model is constructed from a Lego Mindstorms EV3 set, see Figure 5. The model is $21.2 \mathrm{~cm}$ long, 14 $\mathrm{cm}$ wide, and $11.3 \mathrm{~cm}$ high. One EV3 large motor (Figure 6) is connected to the two back wheels, making them run in sync, and one EV3 medium motor (Figure 7) is connected to the steering of the two front wheels. Both are powered/controlled by an EV3 brick (Figure 8). The brick is programmed using the EV3 block-based software where the medium motor's power is manipulated to create the different transitions by turning the front wheels a given angle.

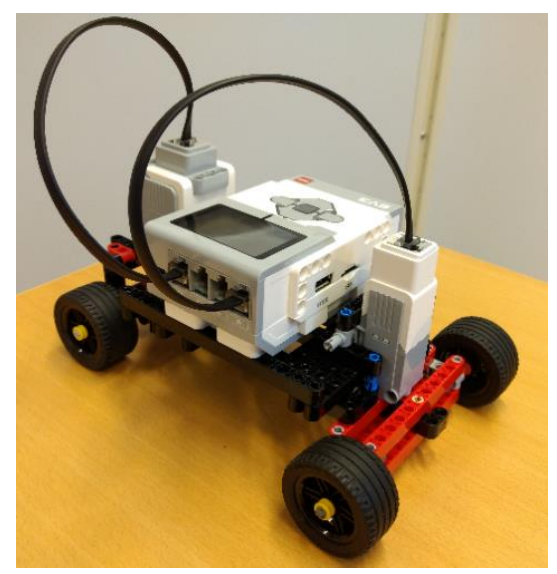

Figure 5. The physical model built with a Lego Mindstorms set.

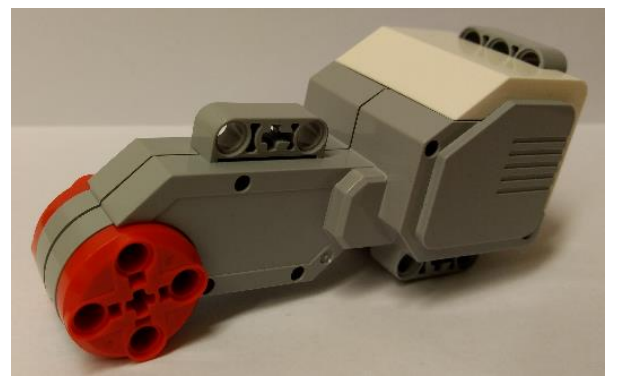

Figure 6. The EV3 Large Motor sensor.

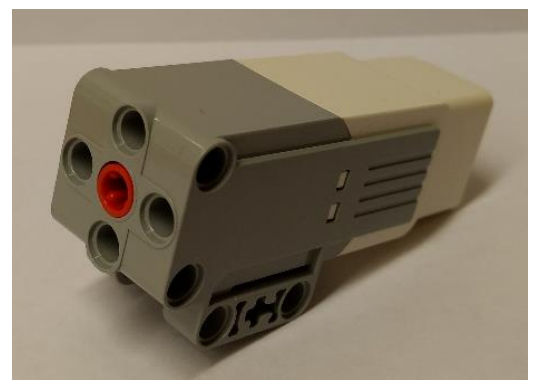

Figure 7. The EV3 Medium Motor sensor. Figure 8. The EV3 intelligent brick.

Table 1 shows the change in motor turn-angle for the three transition cases discretized into 11 steps over 5.5 seconds. A turn-angle of 0 degrees means that the wheels are pointing straight forward making the vehicle drive straight, and a turn-angle above 0 degrees means that the wheels are rotated a given angle, to the left, making the vehicle turn left.

Table 1. Change in motor turn-angle to create different transitions. Discretized into 11 steps over 5.5 seconds.

\begin{tabular}{ccc}
\hline \multicolumn{3}{c}{ Motor turn-angle in degrees for the transition cases } \\
\hline No transition & Clothoid & S-shaped \\
\hline 0 & 0 & 0 \\
0 & 3 & 0.6 \\
0 & 6 & 1.8 \\
0 & 9 & 4.2 \\
0 & 12 & 8.4 \\
0 & 15 & 15 \\
30 & 18 & 21.6 \\
30 & 21 & 25.8 \\
30 & 24 & 28.2 \\
30 & 27 & 29.4 \\
30 & 30 & 30 \\
\hline
\end{tabular}




\section{3-2- The Virtual Model}

The virtual model is created in Blender Game Engine with Bullet as the physics engine, see Figure 9. Compared to the physical model the size of the virtual model is scaled up with a factor of 10 . This is to compensate for the weakness of the physics engine when working with small collision shapes (object sizes should be above 0.2 units [13]). The setup of the vehicle (with physics, engine power, and steering) is done with Python and a Vehicle Controller. The steering values, which controls the angle of the wheels, are given in the same manner as with the Lego vehicle in Table 1, and maximum velocity of the vehicle is set to $3.2 \mathrm{~m} / \mathrm{s}$ (about ten times that of the Lego Mindstorms vehicle).

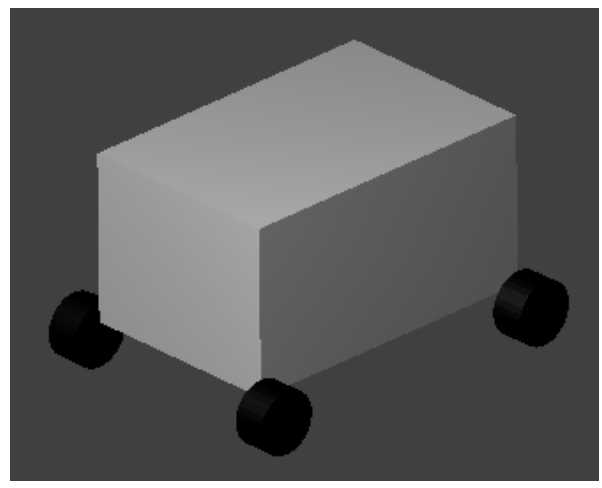

Figure 9. The simple virtual model created in Blender with Bullet physics.

\section{3-3- Experimental Setup}

The setup of the vehicle driving experiments is done as follows: For the Lego model a run lasts 8 seconds going from a straight line to a left-hand turn. The first 1.5 seconds is run with a constant wheel turn-angle of 0 degrees (representing the straight section), then the next 5.5 seconds is run with a wheel angle as described in Table 1 (representing the transition curve), and finally for the last second the turn-angle is held constant at 30 degrees (representing the curved section). The Blender model is based on the same principle as the Lego model, however since the Blender model uses more time than the Lego model to reach maximum speed, the Lego model reaches maximum speed almost instantly, it is run an extra 7.5-8 seconds in the beginning before starting the 8 second run from straight to curved section.

\section{3-4- Analysis}

The test cases are divided into two sections; Geometry tests and VR tests. The geometry tests include testing the transition geometry for the physical model by using a gyroscopic sensor. The sensor measures the rotation rate of the Lego robot in degrees per second and should behave differently for the different transitions. For each transition phase the rotation rate is plotted as a graph, and a visual comparison is made between them. The VR tests include a comparison of the physical model vs the virtual model to look at the accuracy between them. This is done by considering the rotation rate and tracing the paths while driving, then visually comparing the graphs for the two models for each transition case.

\section{4- Results and Discussions}

This section shows and discusses the results from the experiments, with a basis in rotation rate and traced path, by using visual comparison of the plots.

\section{4-1- Geometry Comparison}

In Figure 10 we see the rotation rate of the three transitions for the Lego model. The figure shows a clear distinction between the transitions where the rotation rate has a jump in the no transition case, is linear in the clothoid case, and is smoother for the S-shaped case. The shape of the graphs is comparable to the shape of the curvature in Figure 3, which is to be expected since the lateral forces behave equally to the curvature of the performed curve. This reinforces the credibility of the Lego model as a good test object in spite of its simplicity. The negative graph values just mean that the vehicle is turning left instead of right which would have given equal positive values, and the "jitters" are noise from the sensor. To say something about the observable effects the transitions have on the vehicle we have to look at the spikes in the graphs. The spikes show the moment the wheels are turned and give an indication on how abrupt the change of direction is. The values that are interesting are those moving away from the trend of the graph (the spikes pointing in a positive direction) because they can be interpreted as the force pushing laterally out of the turn. By analysing the spike values in the three graphs it can be seen that the no transition example has the highest value spike with a rotation rate that is approximately $61 \mathrm{deg} / \mathrm{s}$ higher than the average values leading up to the spike. For the two other examples, the clothoid has a maximum spike of $18 \mathrm{deg} / \mathrm{s}$ higher and the $\mathrm{S}$-shape has a maximum of $8 \mathrm{deg} / \mathrm{s}$ higher than the average leading up to the spike. A sudden large jump in lateral forces creates a jerk in the vehicle which can be felt by passengers 
and may lead to a higher need for maintenance in the case of railway tracks, so a smaller jump is more optimal for both passengers, vehicle, and track. From the previous results this indicates that having a transition curve is superior to not having one, and that an S-shaped curvature curve may be preferable over a linear curvature curve, when the jump in rotation rate is the main criterion.
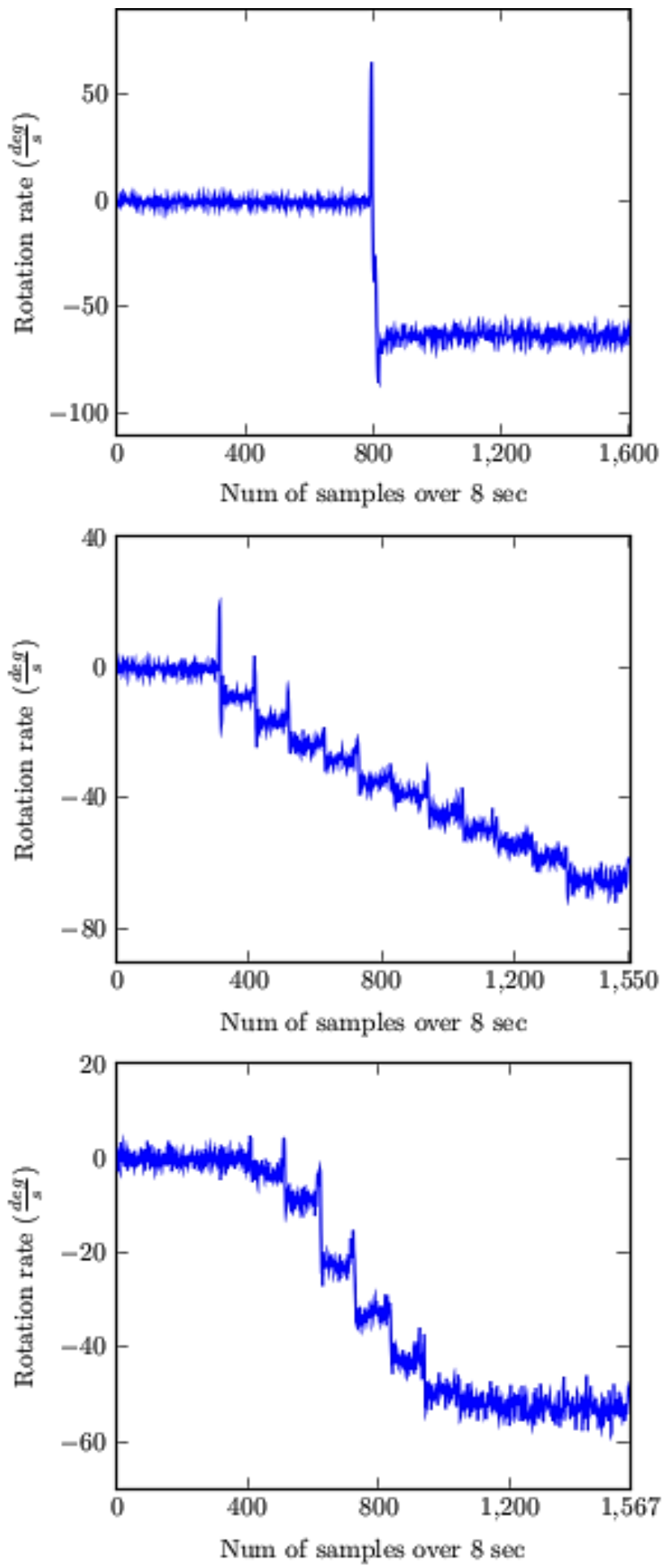

Figure 10. The rotation rate of the three transition cases for the Lego model. Top: no transition; Middle: clothoid transition; Bottom: S-shaped transition.

\section{4-2-Physical vs Virtual Model}

For the comparison of the physical model vs the virtual model we start by looking at the rotation rate. Figure 11 shows the rotation rate of the Blender model, and as described in the previous sub-section Figure 10 shows the rotation rate of the Lego model. When comparing Figure 10 and 11 the biggest difference between them is the lack of noise and spikes in the Blender measurements. The lack of noise comes from the fact that Blender has "perfect" sensors with only 
small numerical errors when measuring rotation rate unlike the Lego model, and the absence of spikes can be explained with insufficient friction between the wheels and ground when turning the wheels. A promising result is that the graphs in the figures have the same shape; a jump in the no transition example, linear in the clothoid example, and S-shaped in the S-shaped example. This shows that the "perfect" Blender model and the "natural" Lego model still behave similar even with simplified Blender physics. If we take a closer look on the Blender graphs, we observe that the jumps between plateaus in the rotation rate are $-36 \mathrm{deg} / \mathrm{s}$ for the no transition case, constantly $-4.5 \mathrm{deg} / \mathrm{s}$ for the clothoid case, and in the $\mathrm{S}$-shaped case it varies from $-0.9 \mathrm{deg} / \mathrm{s}$ to $-10 \mathrm{deg} / \mathrm{s}$, increasing from the smallest jump in the beginning, to the maximum in the middle, then decreasing back to the smallest in the end. Also in this case, as with the spikes in the Lego experiments, a smaller jump is more optimal giving a smoother transition. So a conclusion here is that the clothoid transition is more optimal than no transition, and the S-shaped transition is smoother in the beginning and in the end compared to the clothoid transition, however, for the middle part the clothoid is more optimal. This means that by choosing a transition curve that has a higher continuity curvature in the joints than the linear clothoid, the overall transition will be smoother but the maximum jump in lateral force will be higher. So to obtain an optimal transition curve a proper balance between smoothness and maximum jump of lateral force is essential.
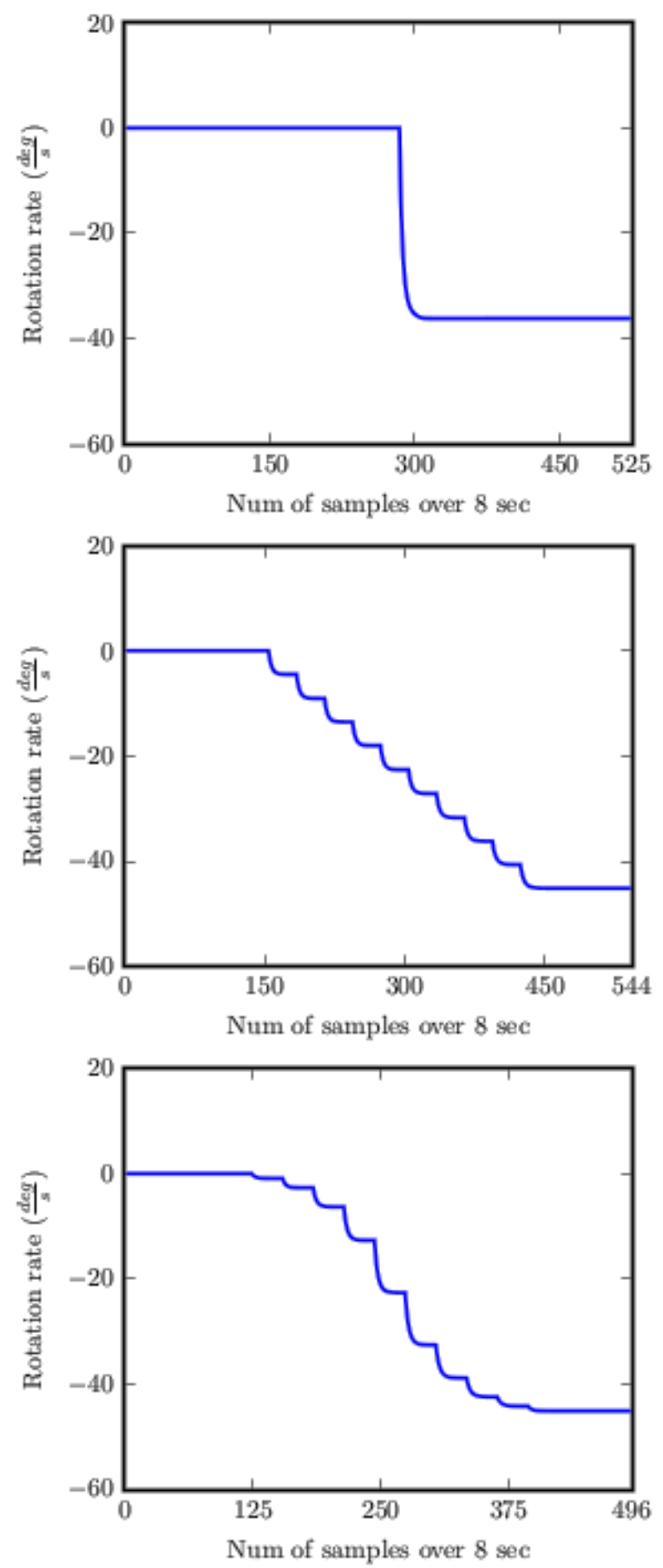

Figure 11. The rotation rate of the three transition cases for the Blender model. Top: no transition; Middle: clothoid transition; Bottom: S-shaped transition. 
The second comparison is done by looking at the traced paths of the models while driving. Figures 12,13 , and 14 show the paths for the three transition cases where the Lego model path is on the top and the Blender model path on the bottom. When comparing them we can clearly see, as expected from the rotation rate comparison, the likeness of the behaviour when driving through the transitions. Each transition example has a characteristic path-shape going into the turn which is clearly observable in both models. The main differences in the graphs are the inability of the Lego model to drive in a perfect straight line, and that the Blender model drives longer and turns wider than the Lego model. The last difference may be due to the wheel-ground friction making it easier for the Blender model to move and turn. Comparing the three transition paths against each other shows that the no transition paths have a distinct spot where the straight segment meets the curved segment, and both the clothoid paths and the S-shaped paths have a more fuzzy transition from the straight segment to the curved segment (which of course makes sense since they have transition curves). The difference between the two last paths is mainly how much they turn when starting with the transition phase, with the S-shaped paths turning more gradual than the clothoid paths.
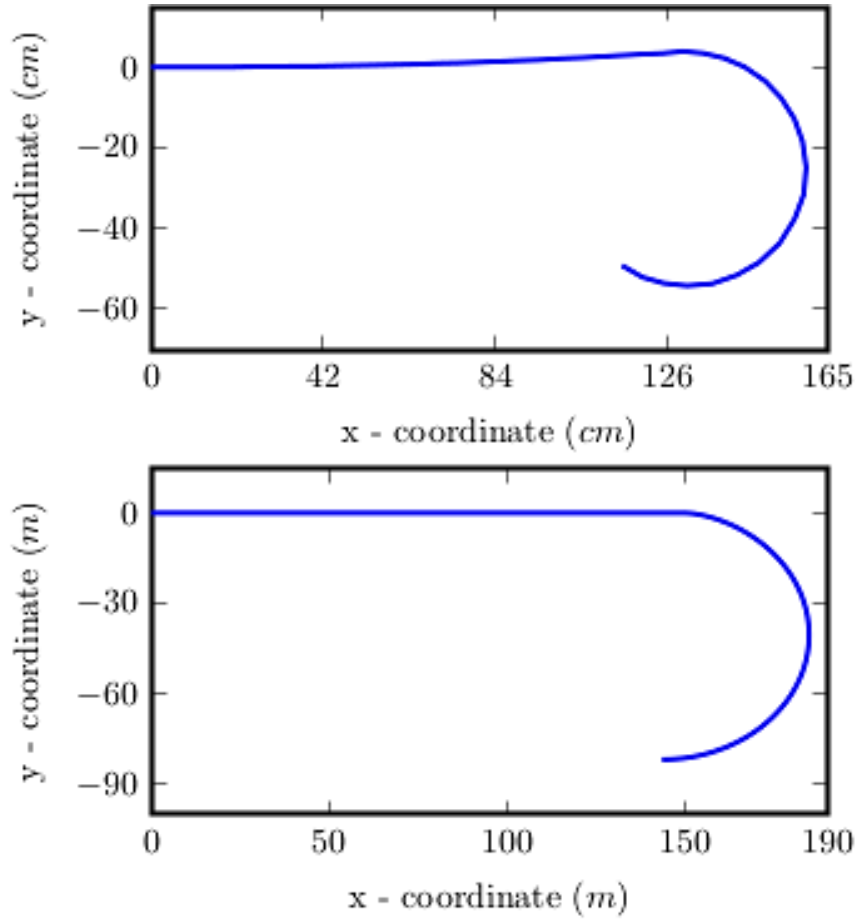

Figure 12. The path traced by the vehicle for the no transition example. Top: Lego model; Bottom: Blender model.
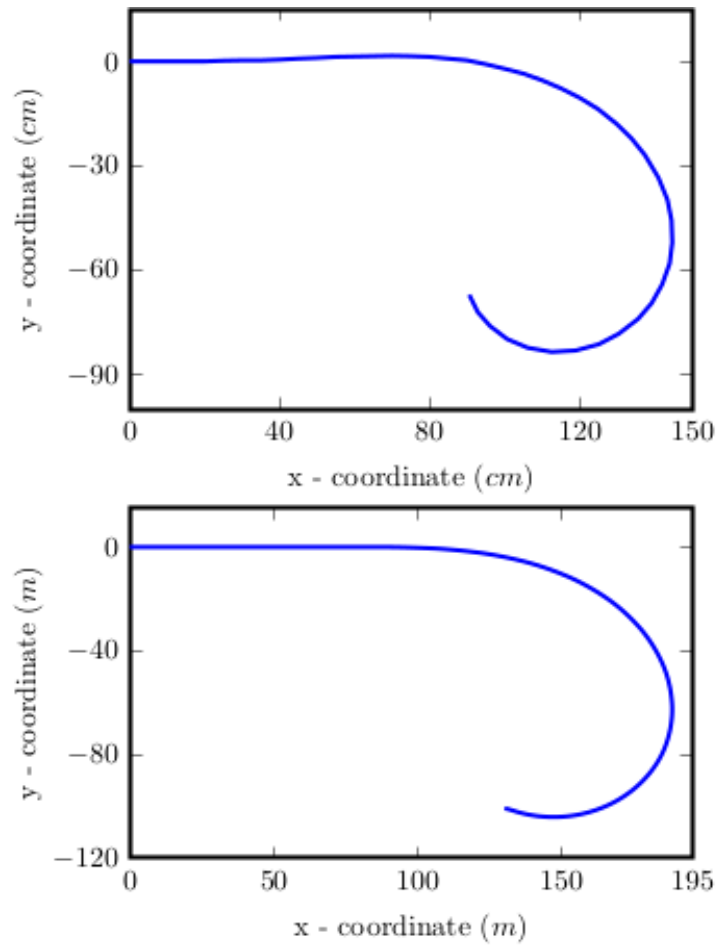

Figure 13. The path traced by the vehicle for the clothoid example. Top: Lego model; Bottom: Blender model. 

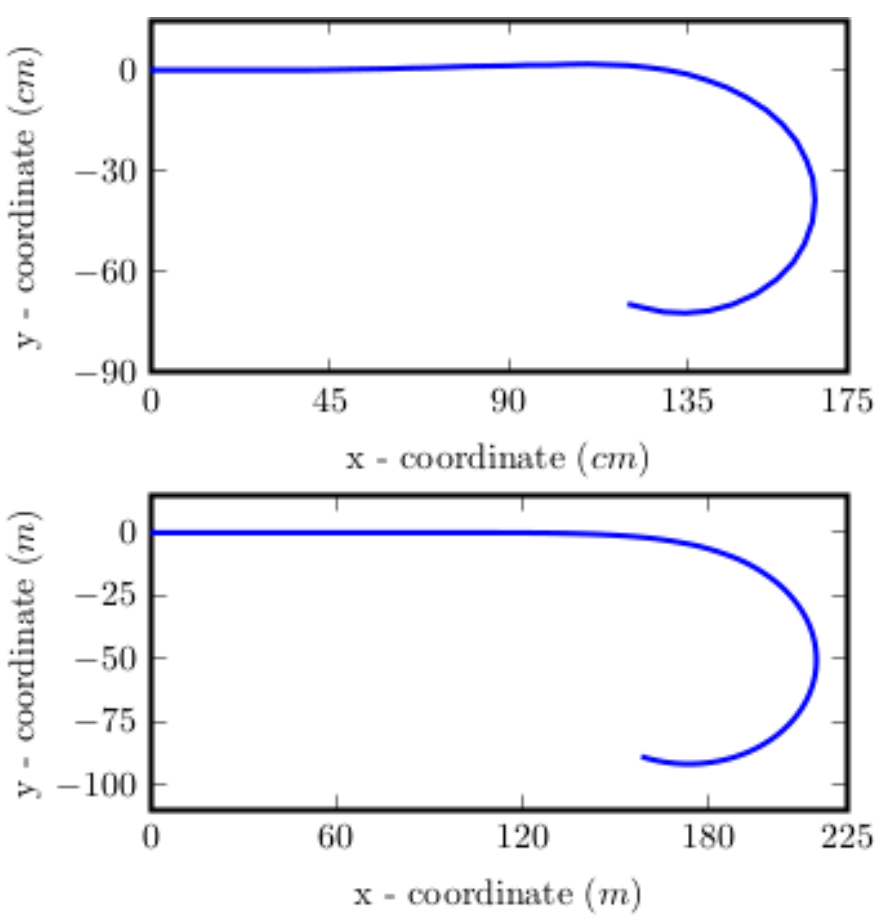

Figure 14. The path traced by the vehicle for the S-shaped example. Top: Lego model; Bottom: Blender model.

\section{5- Conclusion}

In this paper we have looked at a simple Lego vehicle model and used a gyroscopic sensor to examine the effects of transition geometry on the model. In addition we compared the Lego model with a virtual Blender model by looking at rotation rate and path geometry to investigate if a virtual model can give a satisfactory replication of the physical model behaviour. From the gyroscopic measurements of the Lego model we observed that the rotation rate had the same shape as the curvature for each experiment, and that the turning of the wheels created spikes in the graph. These spikes showed the force pushing laterally out of the turn and told us that the no transition turn gave the vehicle a high sudden lateral force compared to the clothoid and S-shaped transitions. When comparing the physical and virtual models we detected that the rotation rates were similar in shape, however the Blender rotation rate did not have any noise or spikes since we were working with a simplified physics model with "perfect" sensors. For the comparison of the traced paths we noticed that the characteristic path-shape of each transition was observable in both models, and that the main difference was connected to distance travelled where the Blender model went a little longer and wider. The results obtained from all experiments tells us that the transition geometry affects the shape of the traced vehicle-path and influence the forces working on the vehicle to a great extent. This means that having a good transition curve is essential in order to minimize the negative effects sudden large changes in force can have on the vehicles, passengers, and tracks (in relation to railway). In addition we conclude that a virtual Blender model gives a good clue on the behaviour of a physical Lego model, with the biggest challenge being wheel-ground friction (especially when turning) and that in some areas (e.g. driving straight) the Blender model is a little too perfect. All in all we think that with small adjustments to the Blender physics a virtual vehicle model can give a satisfactory prediction of the behaviour of a physical vehicle model.

\section{6- Conflict of Interest}

The author declares that there is no conflict of interests regarding the publication of this manuscript. In addition, the ethical issues, including plagiarism, informed consent, misconduct, data fabrication and/or falsification, double publication and/or submission, and redundancies have been completely observed by the authors.

\section{7- References}

[1] Kobryń, Andrzej. "Transition Curves for Highway Geometric Design.” Springer Tracts on Transportation and Traffic (2017). doi:10.1007/978-3-319-53727-6.

[2] Mundrey, J. S. Railway track engineering. Tata McGraw-Hill Education, 2009.

[3] Miller, A. W., M.I.S. (N.S.W.). “The transition spiral.” Australian Surveyor 7, no. 8 (December 1939): 518-526. doi:10.1080/00050326.1939.10436665.

[4] Baykal, Orhan. "Concept of Lateral Change of Acceleration.” Journal of Surveying Engineering 122, no. 3 (August 1996): 132141. doi:10.1061/(asce)0733-9453(1996)122:3(132). 
[5] Baykal, Orhan, Ergin Tari, Zeki Çoşkun, and Muhammed Şahin. "New Transition Curve Joining Two Straight Lines.” Journal of Transportation Engineering 123, no. 5 (September 1997): 337-345. doi:10.1061/(asce)0733-947x(1997)123:5(337).

[6] Tari, E. "The new generation transition curves." ARI the Bulletin of the Istanbul Technical University 54, no. 1 (January 2004): $35-41$.

[7] Tari, Ergin, and Orhan Baykal. "A New Transition Curve with Enhanced Properties.” Canadian Journal of Civil Engineering 32, no. 5 (October 1, 2005): 913-923. doi:10.1139/105-051.

[8] Robertson, G.G., S.K. Card, and J.D. Mackinlay. "Three Views of Virtual Reality: Nonimmersive Virtual Reality.” Computer 26, no. 2 (February 1993): 81. doi:10.1109/2.192002.

[9] Walton, D.J., and D.S. Meek. “A Planar Cubic Bézier Spiral.” Journal of Computational and Applied Mathematics 72, no. 1 (July 1996): 85-100. doi:10.1016/0377-0427(95)00246-4.

[10] Watters, A. "Lego mindstorms: A history of educational robots." Available online: http://hackeducation.com/2015/04/10/mindstorms, (Accessed on 21 June 2019).

[11] Blender. "Blender 2.79 Reference Manual." Available online: https://docs.blender.org/manual/en/latest/index.html, (Accessed on 26 June 2019).

[12] Blain, John M. "The Complete Guide to Blender Graphics” (April 19, 2016). doi:10.1201/b11922.

[13] Coumans, E., R. Ponomarev, J. McCutchan, N. Presson, G. V. den Bergen, C. Ericson, P. Knight et al. "Bullet 2.83 physics SDK manual; 2015.".

[14] Blender. "Vehicle Controller Physics." Available online: https://docs.blender.org/manual/en/2.79/game_engine/physics/types /vehicle.html, (Accessed on 6 August 2019). 